engaging in physical activity seem more achievable for those who are currently inactive. Of note, a recent meta-analysis of 36,383 participants across eight studies similarly reported a steeper dose-response relationship between accelerometer-measured physical activity and mortality risk than that observed in studies using self-reported physical activity as the exposure $^{12}$, so a critical mass of evidence in this area is starting to accumulate.

Overall, the study by Strain et al. ${ }^{2}$ is an important one; its large size and objective measurement of physical activity provides more-robust quantification of the dose-response relationship between physical activity and mortality than has previously been possible. The benefits of physical activity may be greater, and the levels of activity at which they occur may be lower, than previously considered. This could have implications for population-level physical-activity recommendations in the future.

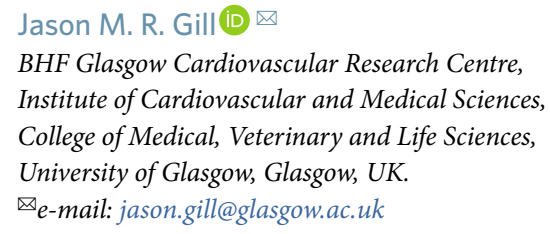
Institute of Cardiovascular and Medical Sciences, College of Medical, Veterinary and Life Sciences, University of Glasgow, Glasgow, UK.

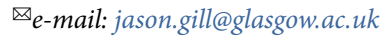

Published online: 17 August 2020 https://doi.org/10.1038/s41591-020-1019-9
References

1. Samitz, G., Egger, M. \& Zwahlen, M. Int. J. Epidemiol. 40, 1382-1400 (2011).

2. Strain, T. et al. Nat. Med. https://doi.org/10.1038/s41591-0201012-3 (2020).

3. Department of Health \& Social Care, Welsh Government, Department of Health Northern Ireland \& Scottish Government. UK Chief Medical Officers' Physical Activity Guidelines (2019).

$\square$ 4. Piercy, K. L. et al. J. Am. Med. Assoc. 320, 2020-2028 (2018).

5. World Health Organization. Global Recommendations on Physical Activity for Health 1-58 (WHO, 2010).

6. Ainsworth, B. E. et al. Med. Sci. Sports Exerc. 43, 1575-1581 (2011)

7. Celis-Morales, C. A. et al. PLoS One 7, e36345 (2012).

8. Allen, N. et al. Health Policy and Technology 1, 123-126 (2012).

9. Moore, S. C. et al. PLoS Med. 9, e1001335 (2012).

10. Kobayashi, L. C., Jackson, S. E., Lee, S. J., Wardle, J. \& Steptoe, A. Age Ageing 46, 427-432 (2017).

11. Strain, T. et al. Int. J. Epidemiol. 49, 162-172 (2020).

12. Ekelund, U. et al. Br. Med. J. 366, 14570 (2019).

Competing interests

The author declares no competing interests.

\title{
Effects of disruption from COVID-19 on antimalarial strategies
}

\author{
Modeling the impact of COVID-19-mitigation strategies on malarial case management and prevention by \\ health services in sub-Saharan Africa predicts 81,000 additional deaths in Nigeria and 769,000 in sub-Saharan
} Africa in 2020.

\section{Rashid Ansumana, Osman Sankoh and Alimuddin Zumla}

$\mathrm{T}$ he spread of COVID-19 across Africa is predicted to increase the existing burden of other major deadly infectious diseases, such as malaria, tuberculosis and AIDS. As of 10 August 2020, there had been 19,462,112 COVID-19 cases globally reported to the World Health Organization (WHO), of which 884,990 cases were from Africa (https://covid19. who.int/). Several African countries have moved into and out of lockdowns, restrictions, border closures and flight disruptions, and there is general panic and apprehension among both urban populations and rural populations. COVID-19 has caused disruptions to health services and a drop in outpatient visits in some places $^{1,2}$, which has affected the extent to which case management of malaria is possible. In this issue of Nature Medicine, Sherrard-Smith et al. describe their modeling study quantifying the potential impact of the spread of COVID-19 on morbidity and mortality from Plasmodium falciparum malaria across sub-Saharan Africa (SSA) and highlight important negative consequences of COVID-19 on malaria-mitigation strategies ${ }^{3}$.

While it is assumed that malaria and COVID-19 do not directly influence the transmission or severity of each other, COVID-19 affects malaria in other ways. In countries in which malaria is endemic, the national infectious-disease response is understandably focused on the COVID-19 pandemic, and there are resulting repercussions on already overstretched and under-resourced health systems. Health systems have become overwhelmed with patients who have COVID-19, and lockdown and other measures introduced to slow transmission interrupt access to routine care and prevention services and medicines. Services such as the widespread use of long-lasting insecticidal nets (LLINs) and indoor residual spraying (which affects vectors of the malaria parasites) are likely to have been interrupted. Furthermore, seasonal malarial chemoprevention and routine case management at all healthcare facilities, such as testing, treating with artemisinin combination therapy and tracking, could have been disrupted.

Compounding these issues, the symptoms of both malaria and COVID-19 include fever, which poses diagnostic and management dilemmas where testing facilities are not optimal.

Sherrard-Smith et al. modeled four different scenarios ${ }^{3}$. First, they modeled unmitigated COVID-19 to illustrate how a rapid epidemic would be highly disruptive to malaria services but for a limited period. Second, they modeled mitigation in which social contact was reduced but the effective reproduction number $(\mathrm{Rt})$ remained above 1, which caused a longer-lasting COVID-19 pandemic. Third, they modeled suppression, in which social distancing reducing $R t$ to $<1$ remained in place until alternative strategies to contain COVID-19 were available, with malaria activities potentially disrupted for a year. Fourth, they modeled suppression lift, in which suppression was sustained but then subsequently lifted, which resulted in a resurgence of the COVID-19 pandemic. Worryingly, they found that all four 


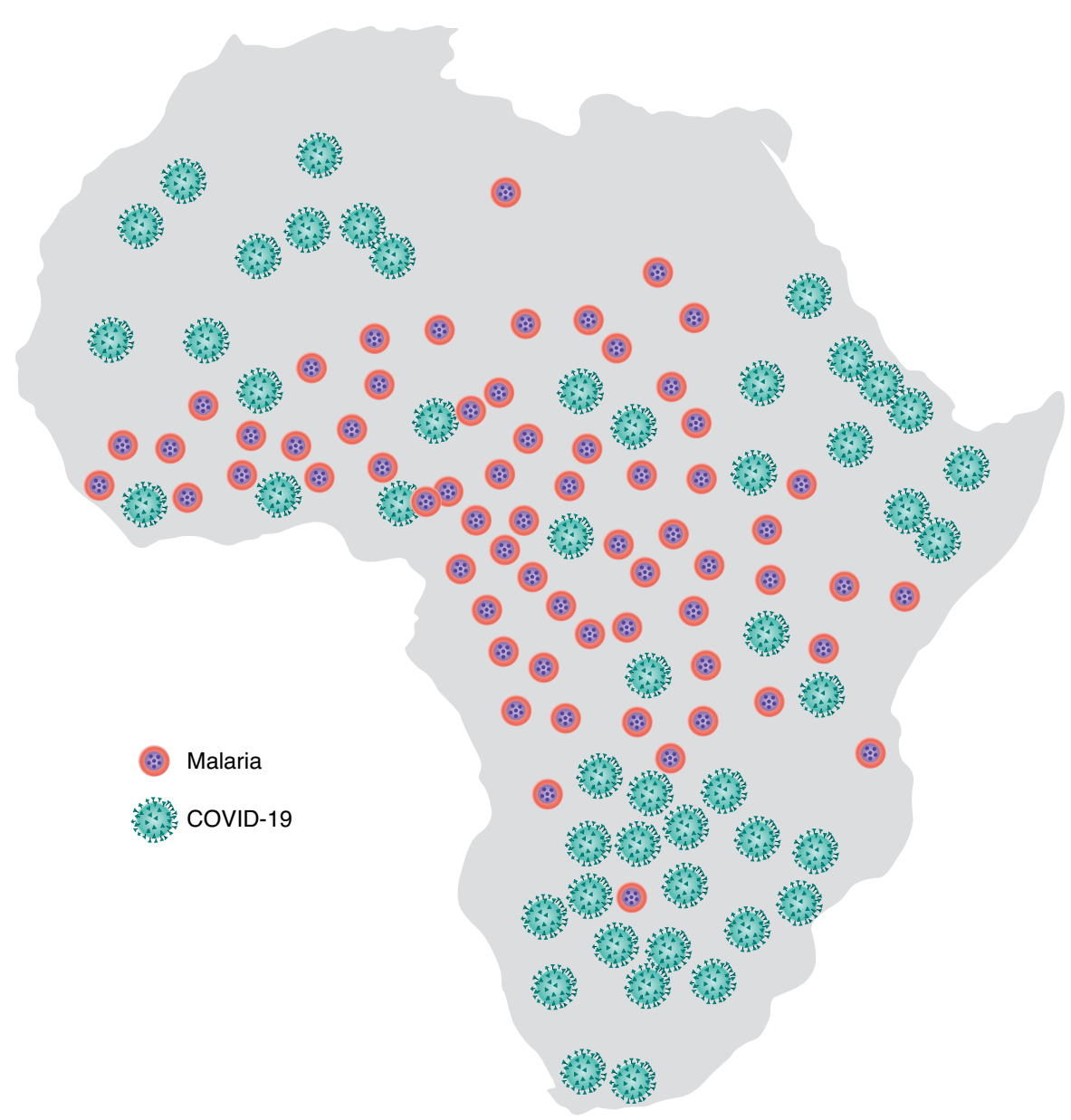

Fig. 1 | Predicted distribution of deaths associated with malaria and COVID-19 in Africa.

COVID-19 scenarios were projected to result in substantial additional deaths from malaria and that while implementing COVID-19-mitigation strategies substantially reduced COVID-19 mortality, the prolonged period of health-system disruption would cause considerably increased malaria deaths.

As with all epidemics and pandemics, COVID-19 has had its own fair share of myths, some of which have affected malaria control adversely or positively. In Sierra Leone, during the distribution of LLINs, there was widespread social-media misinformation that the nets and their use could cause COVID-19 transmission ${ }^{3}$. In addition to the structural challenges noted above, such misinformation could cause reduced uptake and reduced use of the LLINs, with the consequence of reduced vector control and increased transmission events. On the other hand, a bizarre side effect of the misinformation that antimalarial medications are potent against COVID-19 has been an increased uptake of antimalarial drugs and herbs in
SSA, which has potentially caused increased chemoprevention of malaria in adults ${ }^{4,5}$.

The models of Sherrard-Smith et al. ${ }^{1}$ concur with the prediction by the WHO that malaria-related death in SSA could more than double in 2020 because of disruptions in treatment and control programs and that malaria deaths in SSA could reach an estimated 769,000 by the end of 2020 (ref. ${ }^{6}$ ) (Fig. 1). Due to the COVID-19 pandemic, people may be reluctant to seek early diagnosis and treatment for malaria because they are worried about going to clinics, and malaria staff may have been reassigned to work on COVID-19. While African countries have issued recommendations on measures to sustain malaria-control efforts and gains, coordinating provisions for the early diagnosis of malaria in people with fever in endemic areas, and how to differentiate the diagnostic processes for malaria from those for COVID-19 in health services remain challenging ${ }^{7}$. Activities for malaria control that should continue are vector control, continued provision and use of LLINs, indoor residual spraying and chemoprevention for pregnant women and children in targeted communities, and adapted health messages that incorporate physical-distancing guidelines for COVID-19 with activities for malaria education and prevention.

The Ministries of Health and National Malaria Control Programs of all African countries must ensure that activities for the prevention and treatment of malaria continue to be implemented during the COVID-19 era ${ }^{8}$. This will require the National Malaria Control Programs to fully engage with national COVID-19 programs so that testing for both COVID-19 and malaria occurs together; to ensure a continuous supply of, and access to and use of, insecticide-impregnated nets; and to prioritize the areas of highest malaria burden where resources are limited ${ }^{9}$. To prevent the spread of COVID-19 during insecticide spraying and the distribution and delivery of nets, staff should wear face masks, observe physical and social distancing and have regular health checks. Community leaders should be involved in the promotion of behaviors that will prevent malaria transmission and encourage early care-seeking behavior ${ }^{10}$. Potentially contradictory messages must be avoided, and regular educational messages through media, radio and text messaging should be used, inviting the general public to seek early health care.

As an interesting observation from available WHO data on the number of COVID-19 cases and deaths in African countries, it appears that the COVID-19 pandemic has not taken off in Africa as anticipated months ago, especially in high malaria-endemic countries (apart from in South Africa, which is a low malaria zone). Any potentialCOVID-19-malaria interactions in SSA should thus be investigated.

For now, the modeling studies of Sharrard-Smith et al. ${ }^{1}$ remind all to keep our guard up against malaria as well as against COVID-19 to try to avoid excess deaths.

\footnotetext{
Rashid Ansumana (D) 1 凶

Osman Sankoh (1D)1,2,3,4 and Alimuddin Zumla ${ }^{5}$

${ }^{1}$ School of Community Health Sciences, Njala University, Bo, Sierra Leone. ${ }^{2}$ Statistics Sierra Leone, Freetown, Sierra Leone. ${ }^{3}$ School of Public Health, Faculty of Health Sciences, University of the Witwatersrand, Johannesburg, South Africa. ${ }^{4}$ Heidelberg Institute of Global Health, University of Heidelberg Medical School, Heidelberg, Germany. ${ }^{5}$ Center for Clinical Microbiology, Division of Infection \& Immunity, University College London, and UCL Hospitals NIHR Biomedical Research

Centre, London, UK.

$凶_{e-m a i l: \text { r.ansumana@njala.edu.sl }}$
} 
Published online: 17 August 2020

https://doi.org/10.1038/s41591-020-1047-5

References

1. Pallangyo, E., Nakate, M. G., Maina, R. \& Fleming, V. Midwifery 89, 102775 (2020).

2. Chatterji, P. \& Li, Y. National Bureau of Economic Research. http://www.nber.org/papers/w27173.pdf (2020).

3. Sherrard-Smith, E. et al. Nat. Med. https://doi.org/10.1038/ s41591-020-1025-y (2020).

4. Ranasinghe, S. et al. J. Ethnopharmacol. 166, 200-204 (2015).

5. Kapepula, P.M. et al. Am. J. Trop. Med. Hyg. https://doi.

org/10.4269/ajtmh.20-0820 (2020).
6. World Health Organization. https://www.who.int/news-room/ detail/23-04-2020-who-urges-countries-to-move-quickly-to-savelives-from-malaria-in-sub-saharan-africa (2020).

7. Dittrich, S. et al. Lancet Glob. Health 8, e879-e880 (2020).

8. World Health Organization. https://www.who.int/publications/m/ item/tailoring-malaria-interventions-in-the-covid-19-response (2020).

9. Alliance for Malaria Prevention. https://

allianceformalariaprevention.com/wp-content/uploads/2020/ 07/Key-guidance-for-ITN-distribution-during-COVIDtransmission-July-2020.pdf (2020).

10. World Health Organization. https://www.who.int/publications/ i/item/community-based-health-care-including-outreach-andcampaigns-in-the-context-of-the-covid-19-pandemic (2020).
Acknowledgements

R.A. and A.Z. are members of the Pan-African Network on Emerging and Re-emerging Infections (PANDORA-ID-NET) funded by the European and Developing Countries Clinical Trials Partnership the EU Horizon 2020 Framework Programme for Research and Innovation. A.Z. is a UK National Institute for Health Research senior investigator.

Competing interests

The authors declare no competing interests.

\title{
Vaccines targeting SARS-CoV-2 tested in
} humans

\author{
Vaccines directed against SARS-CoV-2 have been administered to healthy volunteers and have been shown to \\ stimulate a brisk humoral and cellular immune response. All vaccines were generally well tolerated with mostly \\ mild to moderate local and systemic reactions.
}

\section{Kathryn M. Edwards}

W ith the escalating numbers of global cases and fatalities associated with the newly discovered coronavirus SARS-CoV-2 (severe acute respiratory syndrome coronavirus 2 ), the recent publication of the results of three phase 1 vaccine trials is welcome news ${ }^{1-3}$. An article by Jackson et al. in the New England Journal of Medicine reports that within only 2 months after the viral sequence of SARS-CoV-2 was published, the first trial participant was given an mRNA vaccine that programmed the synthesis of a pre-fusion spike protein and triggered a brisk immune response ${ }^{1}$. Another report published in Nature highlights the safety and immunogenicity of a different mRNA vaccine $^{2}$, and a recent publication in Lancet describes a third virus-vectored vaccine with reassuring safety and immunogenicity results as well ${ }^{3}$.

The rapid generation of each of these three vaccines was the result of groundwork provided by previous phase 1 studies in humans of vaccines against SARS-CoV-1 and Middle East respiratory syndrome $e^{4,5}$, prior experience with the mRNA and adenovirus-vectored platforms $s^{6,7}$ and the evolution of new vaccine technologies over the past decade in response to other emerging pathogens $s^{8}$.

Jackson et al. report on the safety and immunogenicity of the mRNA vaccine administered to 45 healthy volunteers 18-55 years of age ${ }^{1}$. Two doses of vaccine were administered 1 month apart at three different dose levels, and the responses of the participants to these different regimes were compared. The experimental vaccine was associated with mild to moderate local and systemic adverse events, including local pain and tenderness at the injection site and headache, fever and malaise, with more-severe reactions noted at the highest vaccine dose. Reactions were more common after the second dose than after the first. However, the local and systemic reactions generally were not associated with an inability to continue the functions of everyday life and were self-limited. The authors found that the immune responses raised by participants after the second vaccine dose neutralized SARS-CoV-2 and generated $\mathrm{T}_{\mathrm{H}} 1$ cellular responses, the immune signatures projected to be associated with protection ${ }^{1}$. Since the immune responses to the middle dose and highest dose of vaccine were similar to each other and were greater than responses to the lowest dose, and since the reactogenicity of the highest dose was the greatest, the ongoing efficacy study is using the middle dose.

In the trial published in Nature, a lipid nanoparticle-formulated $\mathrm{mRNA}$ vaccine that encodes the trimerized SARS-CoV-2 spike glycoprotein receptor-binding domain was administered to 45 healthy adults 18-55 years of age who were randomly assigned to receive one of three different vaccine dosages or a placebo and were given two doses of vaccine (or placebo) 21 days apart ${ }^{2}$. Local reactions and systemic events were dose dependent and were generally mild to moderate and self-limited. Antibody concentrations were dose dependent, were boosted by the second vaccine dose and generated functional neutralizing antibody that exceeded levels seen in a panel of COVID-19 convalescent human serum. The efficacy phase of the trial has also begun with an mRNA vaccine that encodes the SARS-CoV-2 full-length spike glycoprotein.

In the phase 1 study in The Lancet, 1,077 participants were randomly assigned to receive either a chimpanzee adenovirus-vectored vaccine expressing the SARS-CoV-2 spike protein or a meningococcal quadrivalent conjugate vaccine $^{3}$. Ten of the participants received a second dose of vaccine. Local and systemic reactions were more common after receipt of the chimpanzee adenovirus spike protein vaccine. Systemic reactions included fever, chills, headache and malaise. Robust humoral and cellular immune responses were seen, and after an additional booster dose, all participants had neutralizing 